\title{
A genetic risk score predicts coronary artery disease in familial hypercholesterolaemia: enhancing the precision of risk assessment
}

Katrina L. Ellis, $\mathrm{PhD}^{\mathrm{a}, \mathrm{b}}$, Amanda J. Hooper, $\mathrm{PhD}^{\mathrm{b}, \mathrm{c}}$, Jing Pang, $\mathrm{PhD}^{\mathrm{b}}$, Dick C Chan, $\mathrm{PhD}^{\mathrm{b}}$, John R. Burnett, MD, PhD ${ }^{\text {b,c,d }}$, Damon A. Bell, PhD ${ }^{\text {b,c,d }}$, Carl J. Schultz, DPhil ${ }^{\text {b,d }}$, Eric K. Moses, PhD ${ }^{\mathrm{a}}$, Gerald F. Watts, MD, DSc $\mathrm{c}^{\mathrm{b}, \mathrm{d}}$

${ }^{\mathrm{a}}$ Centre for Genetic Origins of Health and Disease, School of Biomedical Sciences, University of Western Australia and School of Biomedical Sciences, Curtin University, Perth, Australia

${ }^{\mathrm{b}}$ School of Medicine, Faculty of Medicine and Health Sciences, University of Western Australia, Perth, Australia

${ }^{c}$ Department of Clinical Biochemistry, PathWest Laboratory Medicine WA, Royal Perth Hospital and Fiona Stanley Hospital Network, Perth, Australia

${ }^{\mathrm{d}}$ Department of Cardiology, Royal Perth Hospital, Perth, Australia

\section{Author for correspondence:}

Gerald F. Watts

School of Medicine, Faculty of Medicine and Health Sciences

University of Western Australia

PO Box X2213, Perth, WA 6847, Australia

Tel: +61 8 92249252; Email: gerald.watts@uwa.edu.au

Conflicts of interest: The Authors declare that there is no conflict of interest.

This article has been accepted for publication and undergone full peer review but has not been through the copyediting, typesetting, pagination and proofreading process which may lead to differences between this version and the Version of Record. Please cite this article as doi: $10.1111 /$ cge.13648 


\section{ABSTRACT}

Familial hypercholesterolaemia $(\mathrm{FH})$ is associated with increased risk of coronary artery disease $(\mathrm{CAD})$, however, risk prediction and stratification remains a challenge. Genetic risk scores (GRS) may have utility in identifying FH patients at high CAD risk.

The study included 811 patients attending the lipid disorders clinic at Royal Perth Hospital with mutation-positive $(n=251)$ and mutation-negative $(n=560)$ FH. Patients were genotyped for a GRS previously associated with CAD. Associations between the GRS, clinical characteristics and CAD were assessed using regression analyses.

The average age of patients was 49.6 years and $44.1 \%$ were male. The GRS was associated with increased odds of a $\mathrm{CAD}$ event in mutation-positive $(\mathrm{OR}=3.3 ; 95 \% \mathrm{CI}=1.3-8.2$; $\mathrm{p}=0.009)$ and mutation-negative $\mathrm{FH}$ patients $(\mathrm{OR}=1.8 ; 95 \% \mathrm{CI}=1.0-3.3 ; \mathrm{p}=0.039)$ after adjusting for established predictors of CAD risk. The GRS was associated with greater subclinical atherosclerosis as assessed by coronary artery calcium score $(\mathrm{p}=0.039)$.

A high GRS was associated with CAD defined clinically and angiographically in FH patients. High GRS patients may benefit from more intensive management including lifestyle modification and aggressive lipid-lowering therapy. Further assessment of the utility of the GRS requires investigation in prospective cohorts, including its role in influencing the management of $\mathrm{FH}$ patients in the clinic.

Key words: Genetic risk score; coronary artery disease; familial hypercholesterolaemia; atherosclerosis 


\section{INTRODUCTION}

Familial hypercholesterolaemia (FH) is an autosomal co-dominant disorder characterised by markedly elevated low density lipoprotein cholesterol (LDL-c) and a substantially increased risk of developing coronary artery disease $(\mathrm{CAD})$ at an early age, affecting $~ 1$ in 300 individuals worldwide. Most cases of molecularly defined FH result from pathogenic mutations in the LDLR ( 95\%), APOB (3-5\%) and PCSK9 (1-3\%) genes. ${ }^{1}$ Phenotypic diagnostic tools, such as the Dutch Lipid Clinic Network criteria, are also widely employed in the diagnosis of FH. However, while genetic testing remains the most accurate approach for diagnosing $\mathrm{FH}$, an underlying molecular cause is not identified in 40 to 60 percent of individuals diagnosed with FH using established clinical criteria. ${ }^{2,3}$ Patients without pathogenic FH causing variants are likely have a polygenic basis to their hypercholesterolaemia, or possibly a currently unidentified pathogenic $\mathrm{FH}$-causing mutation. ${ }^{4}$ Individuals with pathogenic FH-causing variants have been shown to be at an increased risk of CAD when compared with individuals with the same LDL-c levels, but without a pathogenic variant. ${ }^{5}$ Nevertheless, even in individuals carrying the same pathogenic $\mathrm{FH}$ variant there is variability in CAD risk, and risk stratification and prediction remains a challenge. Although risk equations including traditional CAD risk factors have been developed and show utility in patients with $\mathrm{FH}$, risk stratification remains imperfect and further work to refine CAD risk prediction is required. Recently, several genetic risk scores (GRS) for CAD, which include tens to millions of single nucleotide polymorphisms (SNPs), have been associated with CAD events in large case-control and prospective investigations. ${ }^{6-9}$ Furthermore, a 50-variant GRS has been shown to improve CAD discrimination and reclassification in 23,595 participants from the Malmo Diet and Cancer study, independently of self-reported family history. ${ }^{6}$ 
Precision medicine, in which genomics plays a crucial role, is important for ensuring the right treatment for the right patient at the right time, even in monogenic disorders like FH. It is also important for ensuring treatment is more cost-effective. We hypothesised that a GRS for CAD may contribute to the identification of FH patients at increased cardiovascular risk. Patients deemed to be at high cardiovascular risk may benefit from more intensive interventions including earlier CAD risk assessment with CT-coronary angiogram, and more aggressive lipid-lowering therapy that incorporates early initiation of PCSK9 inhibitors and other lipid-lowering agents.

\section{METHODS}

\section{Study population}

Study participants included 811 patients with suspected FH, defined by a Dutch Lipid Clinic Network Criteria Score of $\geq 3$ corresponding to at least possible FH. Patients were screened for FH at the Lipid Disorders Clinic at Royal Perth Hospital after referral from general practice, coronary care, or ambulatory specialist care. As part of a dedicated diagnostic service, genetic testing for known pathogenic $\mathrm{FH}$-causing variants within genes encoding $L D L R, A P O B$ and $P C S K 9$ was carried out as previously reported. ${ }^{2}$ The final study consisted of 251 'mutation-positive' index cases in whom a pathogenic variant was identified with DNA testing and 560 'mutation-negative' index cases in whom an FH-causing variant could not be found.

Each patient provided written informed consent, and human ethics approval was granted by the Royal Perth Hospital Ethics Committee (EC 2016/155), and the Human Research Ethics Office of the University of Western Australia (RA/4/1/8669). 


\section{Clinical characteristics}

Each patient had standard clinical and anthropometric characteristics recorded including medical and social history, current medications, body mass index (BMI) and blood pressure. Personal history of CAD was additionally documented. A positive history of CAD was documented from patient clinical notes and defined as previous myocardial infarction (MI), coronary angioplasty or stenting, coronary artery bypass grafting, or angina pectoris. As part of standard clinical care, coronary artery calcium scores were calculated for 268 patients without a documented history of CAD from contrast enhanced CT coronary angiograms using a modified agatston score. ${ }^{10}$.

\section{Genetic and Biochemical Analyses}

Genomic DNA was extracted from peripheral blood leukocytes using the Wizard ${ }^{\circledR}$ Genomic DNA Purification Kit (Promega, Madison, WI, USA). Genotyping was carried out using the SEQUENOM platform for 50 SNPs included in GRS for coronary heart disease (CHD) previosuly reported by Tada et.al. ${ }^{6}$ Of the 50 SNPs submitted, 44 were successfully genotyped (Supplementary Table 1); reasons for SNP failure included an inability to amplify the region surrounding the SNP and poor clustering. The genotyping success rate for the final 44 SNPs was $>95 \%$.

Plasma total cholesterol, high-density lipoprotein cholesterol (HDL-c), and triglyceride concentrations were measured at National Association of Testing Authorities-accredited laboratories employing standard enzymatic and immunoturbidimetric methods. LDL-c was calculated using the Friedewald equation ${ }^{11}$ except for patients where triglyceride levels were between 4.5 and $10.0 \mathrm{mmol} / \mathrm{L}$; who had direct measurement of LDL-c. Lipoprotein (a) [Lp(a)] was measured with an latex-enhanced immunoassay considered to be independent of 
apo(a) isoform size [Quantia Lp(a) assay and standard] using the Abbott Architect c16000 platform (Abbott Laboratories, Abbott Park, IL).

\section{Statistical Analysis}

For each patient, the CAD GRS was calculated as follows: for each SNP the previously reported risk estimate (Supplementary Table 1) was natural log transformed and multiplied by one for patients that were heterozygous and two for those that were homozygous for the modelled allele. These products were then summed across the 44 SNPs included in the GRS. The association between GRS and clinical and anthropometric characteristics was assessed using linear and logistic regression as appropriate. $L D L R$ variants were classified as activitydefective or activity-negative ('null') according to variant type; nonsense, frameshift and copy number variants were classed as 'null' and other variants classed as 'defective' unless functional studies have demonstrated otherwise. ${ }^{12,13}$ The association between GRS and CAD was investigated using logistic regression analyses that adjusted for the established predictors of increased CAD risk: age, sex, hypertension, type-2 diabetes, smoking status, $L p(a)$ levels and treatment-adjusted LDL-c. The association between GRS and coronary calcium score was investigated using linear regression adjusting for the same established predictors of CAD. Due to a smaller sample size for the coronary calcium score analyses, FH mutation positive and negative individuals were analysed together with the presence of pathogenic $\mathrm{FH}$ mutation included as an additional variable in the model. To account for the possibility of having a calcium score of 0 , a value of 1 was added to each individuals score prior to carrying out a natural log transformation. In patients receiving lipid-lowering therapy at the time of study enrolment, treatment-adjusted LDL-c was estimated by applying a correction factor reflecting previously described effects of statins and/or ezetimibe therapy. ${ }^{14}$ All 
analyses were carried out in SPSS Statistics version 25. Statistical significance was reached if $\mathrm{p} \leq 0.05$.

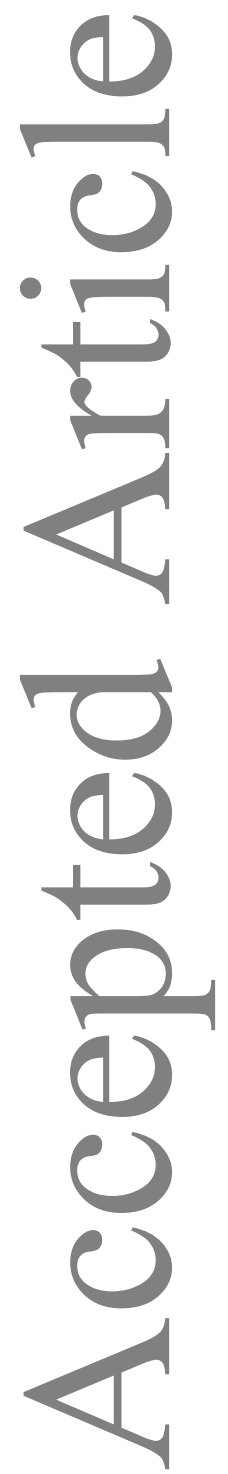




\section{RESULTS}

\section{Baseline patient characteristics}

The baseline clinical characteristics of mutation-positive and mutation-negative $\mathrm{FH}$ patients are shown in Table 1. Mutation-positive FH patients were on average younger when they entered the clinic (45.4 vs. 51.4 years; $\mathrm{p}<0.001)$ and had a higher (LDL-c (8.0 vs. 6.0 $\mathrm{mmol} / \mathrm{L} ; \mathrm{p}<0.001)$, but lower triglyceride (1.2 vs. $1.6 \mathrm{mmol} / \mathrm{L} ; \mathrm{p}<0.001)$ levels and a lower BMI (27.1 vs $\left.28.2 \mathrm{~kg} / \mathrm{m}^{2} ; \mathrm{p}=0.007\right)$ when compared with mutation-negative FH patients. Additionally, fewer patients with mutation-positive FH had a history of hypertension (23.1 vs. $33.2 \%$; $=0.004)$, and a greater proportion were receiving statin therapy at their baseline clinic visit (70.5 vs. $56.3 \%$; $\mathrm{p}<0.001)$ than those with mutation-negative FH. There was no significant difference in mean age of first CAD event when comparing patients with and without an FH mutation ( $46 \pm 9.5$ vs. $48.6 \pm 10.9$ years, $\mathrm{p}=0.176)$.

\section{GRS and clinical characteristics}

The mean CAD GRS was slightly higher for mutation-negative FH patients when compared with mutation-positive FH patients (GRS $3.69 \pm 0.43$ vs. $3.56 \pm 0.41 ; \mathrm{p}<0.001$; Figure 1), suggesting the referral bias of our clinic population. There was no significant difference in mean GRS when comparing patients of caucasian ancestry with those of non-caucasian ancestry (GRS 3.64 \pm 0.42 vs $3.71 \pm 0.47 ; \mathrm{p}=0.139)$. The mean CAD GRS was also not significantly different in patients with family history of CAD (presence vs. absence $3.67 \pm 0.43$ vs $3.65 \pm 0.42, p=0.666$ ), gender with age $<45$ years old (male vs. female $3.58 \pm 0.42$ vs. $3.62 \pm 0.44, \mathrm{p}=0.549$ ), gender with age $\geq 45$ years old (male vs. female $3.71 \pm 0.43$ vs. $3.65 \pm 0.41, \mathrm{p}=0.113$ ) and class of $L D L R$ mutation variants (null vs. defective $3.56 \pm 0.41$ vs. $3.59 \pm 0.42, \mathrm{p}=0.648)$. There was also no significant difference in proportion of CAD $(25 \%$ vs. 
26\%, $\mathrm{p}=0.871$ ) between FH patients with $L D L R$ null and defective mutations. In the overall FH cohort, regression analyses revealed a significant positive association between GRS and hypertension $(\mathrm{OR}=2.0 ; \mathrm{p}<0.001)$ and $\mathrm{Lp}(\mathrm{a})$ levels $(\beta=0.74 ; \mathrm{p}<0.001)$. Conversely, a negative association was observed with treatment-adjusted LDL-c $(\beta=-0.31 ; \mathrm{P}=0.025)$, potentially reflecting the higher polygenic risk score in mutation-negative FH patients whom on average have lower LDL-c levels (Table 2).

Association between GRS and CAD At the time of study enrolment, 203 (25.1\%) of patients had experienced a CAD event. This included 63 (25.1\%) mutation-positive FH patients, and $140(25.0 \%)$ mutation-negative $\mathrm{FH}$ patients. Univariate analysis identified a significant association between GRS and CAD events in mutation-positive FH patients $(\mathrm{OR}=2.9 ; 95 \% \mathrm{CI}=1.4-6.0 ; \mathrm{p}=0.004)$. This association was independent of type of $L D L R$ mutation (null vs. defective mutation) with a 2.3 increased odds of CAD in these patients $(\mathrm{CI}=1.09-4.89 ; \mathrm{p}=0.029)$. Likewise, there are a significant association between GRS and CAD events in mutation-negative patients $(\mathrm{OR}=2.97 ; 95 \% \mathrm{CI}=1.86-4.75 ; \mathrm{p}<0.001)$. The significant association between GRS and CAD events remained statistically significant when adjusting for established predictors of increased CAD risk, with GRS associated with a 3.31 increased odds of CAD in mutation-positive FH (Table 3) and a 1.83 increased odds of CAD in mutation-negative FH patients (Table 4). The association with CAD was also independent of ethnicity, the presence of tendon xanthomas, and of self reported family history of premature $\mathrm{CAD}$ in a subset of patients $(\mathrm{n}=338)$ in which a detailed family history was collected (Supplementary Table 2). Furthermore, in mutation-positive FH the association between GRS and CAD was independent of the Montreal $(\mathrm{p}=0.005$; Supplementary Table 3) and modified SAFEHEART ( $p=0.009$; Supplementary Tables 4 and 5) FH risk equations. 


\section{Association between GRS and coronary calcium score}

Univariate analysis revealed a trend between the CAD GRS and coronary calcium score in 268 CAD event-free individuals that had undergone CT-coronary angiography as part of routine clinical care $(\beta=0.59 ; 95 \% \mathrm{CI}=-0.07-1.26 ; \mathrm{p}=0.081)$. However, the association between GRS and a higher coronary calcium score was significant when adjusting for established predictors of increased CAD risk, including the presence of a pathogenic FH mutation $(\beta=0.67 ; 95 \% \mathrm{CI}=0.03-1.31 ; \mathrm{p}=0.039$; Table 5). 


\section{DISCUSSION}

We have identified a novel and independent association between a CAD GRS, previously associated with CAD in a large population study ${ }^{6}$, and an increased odds of CAD defined clinically and angiographically in patients with both molecularly and phenotypically defined FH. The findings provide and exemplar of the potential of genetic testing in refining risk prediction in a disorder with wide variability in predisposition to CAD.

The utility of polygenic risk scores for stratifying CAD risk in patients with FH beyond traditional risk factors is supported by recent findings from the Montreal Clinic Research Institute, in which a 192 SNP GRS also independently predicted the prevalence of cardiovascular disease in patients with genetically-defined $\mathrm{FH} .{ }^{15}$ It is worth noting that all participants from the Montreal Clinic were of French-Canadian origin and screened for the presence of classical French-Canadian mutations in the $L D L R$ gene; the FH mutations reported in those studies differs substantially from the mutation spectrum observed in our study of an Australian population.

Risk stratification and prediction in patients with FH remains an important challenge with variable risk often observed in patients carrying the same pathogenic FH mutation. Although, conventional risk prediction tools such as the Framingham and Pooled Cohort equations have established utility in the general population they are not designed to be applied in patients with FH. Recent advances have been made with the development of FH specific risk prediction models, with the SAFEHEART Risk Equation (SAFEHEART-Re) ${ }^{16}$ associated with a Harrell C index of 0.81 and the Montreal-FH-SCORE (MFHS) ${ }^{17}$ giving an area under the curve (AUC) of 0.799 . Nevertheless, residual risk remains which may be explained by the interaction of multiple genetic and environmental factors. In the present study, we identified that the CAD GRS was associated with the presence of CAD independent of both the 
SAFEHEART and Montreal FH risk equations in patients with genetically confirmed FH, however, whether adding the GRS to these FH risk equations incrementally improves risk prediction needs to be tested longitudinally in studies with prospective patient outcome data.

There have been a few additional published investigations on the impact of common genetic variation on CAD risk in patients with FH. In the Spanish SAFEHEART registry, polymorphisms in the VEGFR and $O P G$ genes which encode vascular endothelial growth factor and osteoprotegerin, respectively, were associated with the accumulation of coronary calcium as measured with coronary tomographic angiography. ${ }^{18}$ There have also been several separate reports from the Montreal Clinic Research Institute that have demonstrated associations between SNPs within $S L C 22 A 3^{19}, A B O^{20},{ }^{2} C S K 9^{21}, L O X 1^{22}, P H A C T R 1^{23}$, $L P A^{24}$, and chromosome 9p21.3 locus ${ }^{25}$ with the presence of cardiovascular disease. GRS for LDL-c and triglycerides have also been proposed to distinguish FH patients with an underlying monogenic or polygenic cause of their hypercholesterolaemia., 26,27

While all patients with suspected FH should be encouraged to follow a healthy lifestyle, adherence to lifestyle program is universally disappointing. Information regarding hidden, inherited risk of CAD (e.g. a high GRS) may assist clinicians and patients in making better shared-decisions on treatment; it could encourage patients to more closely adhere to lifestyle modifications and medical treatments, potentially improving CAD outcomes. However, the clinical utility of a GRS in the management of patients with FH remains to be demonstrated. In a study involving four prospective cohorts totaling 55,685 participants, a high polygenic risk score and lifestyle factors were independently associated with an increased incidence of coronary events. ${ }^{28}$ That study also demonstrated that among patients classified as being at high genetic risk of CAD, a favourable lifestyle conferred a nearly $50 \%$ lower risk of CAD than an unfavourable lifestyle, highlighting the importance of lifestyle intervention in 
individuals with a high genetic burden for $\mathrm{CAD} .{ }^{28} \mathrm{~A}$ study carried out in participants from a randomised controlled trial of primary prevention with statin therapy, and two observational cohort studies, identified that patients with a high GRS for CAD had a greater burden of subclinical atherosclerosis, but derived both greater relative and absolute benefit from statin therapy to prevent first CAD events. ${ }^{29}$ Specifically, in patients with a high GRS, the relative risk reduction with statin therapy was $46 \%$ compared with $26 \%$ for all other patients, with the absolute risk reduction $3.6 \%$ versus $1.3 \%$, respectively. ${ }^{29}$ These results are supported by a study carried out by Mega $e t . a l^{30}$ involving a community based cohort study and four randomised controlled trials of primary and secondary prevention. They identified a gradient of increasing risk for incident and recurrent CHD with a CHD GRS, and demonstrated a gradient of increasing relative risk reduction with statin therapy across increasing GRS categories. Furthermore, the number needed to treat to prevent one event was lower in those at high genetic risk. ${ }^{30,31}$ Taken in combination, these findings suggest that patients with FH who are also at high genetic risk for CAD may derive particular benefit from intensive lifestyle management, early assessment of CAD risk (e.g. coronary artery calcium scoring) and aggressive initiation of statin therapy and other therapeutic agents, for example PCSK9 inhibitors. However, this requires further investigation in large prospective cohorts of $\mathrm{FH}$ patients.

There are several strengths of the present study. The cohort reported is clinically well characterised and included both molecularly and phenotypically defined FH patients. Furthermore, the FH mutation-positive group incorporated a broad range of pathogenic mutations within the LDLR, APOB and PCSK9 genes, indicating the generalizability of the findings in FH patients carrying these mutations. The main limitation of the study was the lack of prospective follow-up data and the diffuse CAD event outcome phenotype employed. The GRS was also developed in patients of predominantly European ancestry, with the 
present study also a mostly Caucasian population. Hence the utility of the score in nonCaucasian FH patients, where resources are often more scarce, requires investigation. Finally, the reduced number of gene variants (44 as opposed to 50) included in the calculation of the GRS may have impacted on the strength of associations observed in the study, however, any effect is likely to have been modest.

There are also a number of practical implications of translating the GRS into clinical practice in patients with FH. Although testing for the GRS is safe, and could readily be incorporated into routine FH genetic testing platforms at little extra cost, additional genetic counselling may be required, including in women planning pregnancy. In young $\mathrm{FH}$ patients with a high GRS, treatment may initally focus on the management of lifestyle factors with particular attention to diet and exercise, smoking cessation, and the prevention of diabetes and hypertension. In adult patients, management may involve the use of additional agents for LDL-c lowering, initation of therapies for reducing triglyceride-rich lipoproteins, antiinflammatory agents, anti-coagulants, and in the future therapies aimed at lowering $\operatorname{Lp}(\mathrm{a})$. In conclusion, a GRS was associated with prevalent CAD in a clinic population with both mutation-positive and mutation-negative FH. This finding was further supported by an association with greater subclinical atherosclerosis, as assessed with coronary artery calcification. Although these associations were independent of traditional CAD risk factors and FH risk algorithms, the clinical utility of the GRS in risk prediction and stratification requires further investigation in large populations of $\mathrm{FH}$ patients with prospective follow-up data. There is also no evidence that the use of a GRS influences the management of patients and is cost-effective. Hence, the value of the GRS in modifying management decisions in FH will need to be demonstrated before its incorporation into routine clinical practice. 


\section{ACKNOWLEDGEMENTS}

KLE was supported by a Royal Perth Hospital Medical Research Foundation Fellowship and a Raine Medical Research Foundation grant. JP was supported by a WAHTN Early Career Fellowship and the Australian Government's Medical Research Future Fund.

\section{DATA AVAILABILITY STATEMENT}

The data that support the findings of this study are available from the corresponding author upon reasonable request.

\section{REFERENCES}

1. Nordestgaard BG, Chapman MJ, Humphries SE, et al. Familial hypercholesterolaemia is underdiagnosed and undertreated in the general population: guidance for clinicians to prevent coronary heart disease: consensus statement of the European Atherosclerosis Society. Eur Heart J 2013; 34: 3478-3490a. DOI: 10.1093/eurheartj/eht273.

2. Hooper AJ, Nguyen LT, Burnett JR, et al. Genetic analysis of familial hypercholesterolaemia in Western Australia. Atherosclerosis 2012; 224: 430-434.

3. Talmud PJ, Shah S, Whittall R, et al. Use of low-density lipoprotein cholesterol gene score to distinguish patients with polygenic and monogenic familial hypercholesterolaemia: a case-control study. Lancet 2013; 381: 1293-1301. DOI: 10.1016/S0140-6736(12)62127-8. 4. Ellis KL, Hooper AJ, Burnett JR, et al. Progress in the care of common inherited atherogenic disorders of apolipoprotein B metabolism. Nat Rev Endocrinol 2016; 12: 467484. Review. DOI: 10.1038/nrendo.2016.69 
http://www.nature.com/nrendo/journal/v12/n8/abs/nrendo.2016.69.html\#supplementaryinformation.

5. Khera AV, Won HH, Peloso GM, et al. Diagnostic Yield and Clinical Utility of Sequencing Familial Hypercholesterolemia Genes in Patients With Severe Hypercholesterolemia. J Am Coll Cardiol 2016; 67: 2578-2589. DOI:

10.1016/j.jacc.2016.03.520.

6. Tada H, Melander O, Louie JZ, et al. Risk prediction by genetic risk scores for coronary heart disease is independent of self-reported family history. Eur Heart J 2016; 37: 561-567. 2015/09/24. DOI: 10.1093/eurheartj/ehv462.

7. Khera AV, Chaffin M, Aragam KG, et al. Genome-wide polygenic scores for common diseases identify individuals with risk equivalent to monogenic mutations. Nature genetics 2018; 50: 1219.

8. Inouye M, Abraham G, Nelson CP, et al. Genomic Risk Prediction of Coronary Artery Disease in 480,000 Adults. Implications for Primary Prevention 2018; 72: 1883-1893. DOI: 10.1016/j.jacc.2018.07.079.

9. Krarup NT, Borglykke A, Allin KH, et al. A genetic risk score of 45 coronary artery disease risk variants associates with increased risk of myocardial infarction in 6041 Danish individuals. Atherosclerosis 2015; 240: 305-310. DOI:

https://doi.org/10.1016/j.atherosclerosis.2015.03.022.

10. Agatston AS, Janowitz WR, Hildner FJ, et al. Quantification of coronary artery calcium using ultrafast computed tomography. J Am Coll Cardiol 1990; 15: 827-832. 1990/03/15. DOI: 10.1016/0735-1097(90)90282-t.

11. Friedewald WT, Levy RI and Fredrickson DS. Estimation of the concentration of lowdensity lipoprotein cholesterol in plasma, without use of the preparative ultracentrifuge.

Clinical chemistry 1972; 18: 499-502. 
12. Rodríguez-Jiménez C, Pernía O, Mostaza J, et al. Functional analysis of new variants at the low-density lipoprotein receptor associated with familial hypercholesterolemia. Hum Mutat 2019; 40: 1181-1190. doi: 10.1002/humu.23801.

13. Hobbs HH, Brown MS, Goldstein JL. Molecular genetics of the LDL receptor gene in familial hypercholesterolemia. Hum Mutat 1992;1:445-466.

https://doi.org/10.1002/humu.1380010602

14. Haralambos K, Whatley SD, Edwards R, et al. Clinical experience of scoring criteria for Familial Hypercholesterolaemia (FH) genetic testing in Wales. Atherosclerosis 2015; 240: 190-196. DOI: https://doi.org/10.1016/j.atherosclerosis.2015.03.003.

15. Paquette M, Chong M, Theriault S, et al. Polygenic risk score predicts prevalence of cardiovascular disease in patients with familial hypercholesterolemia. J Clin Lipidol 2017; 11: 725-732 e725. 2017/05/01. DOI: 10.1016/j.jacl.2017.03.019.

16. Perez de Isla L, Alonso R, Mata N, et al. Predicting Cardiovascular Events in Familial Hypercholesterolemia: The SAFEHEART Registry (Spanish Familial Hypercholesterolemia Cohort Study). Circulation 2017; 135: 2133-2144. 2017/03/10. DOI:

\subsection{1/CIRCULATIONAHA.116.024541.}

17. Paquette M, Brisson D, Dufour R, et al. Cardiovascular disease in familial hypercholesterolemia: Validation and refinement of the Montreal-FH-SCORE. J Clin Lipidol 2017; 11: 1161-1167 e1163. 2017/08/13. DOI: 10.1016/j.jacl.2017.07.008.

18. Miramontes-Gonzalez JP, Usategui-Martin R, Perez de Isla L, et al. VEGFR2 and OPG genes modify the risk of subclinical coronary atherosclerosis in patients with familial hypercholesterolemia. Atherosclerosis 2019; 285: 17-22. 2019/04/17. DOI:

10.1016/j.atherosclerosis.2019.03.019. 
19. Paquette M, Bernard S and Baass A. SLC22A3 is associated with lipoprotein (a) concentration and cardiovascular disease in familial hypercholesterolemia. Clin Biochem 2019; 66: 44-48. 2019/02/18. DOI: 10.1016/j.clinbiochem.2019.02.008.

20. Paquette M, Dufour R and Baass A. ABO blood group is a cardiovascular risk factor in patients with familial hypercholesterolemia. J Clin Lipidol 2018; 12: 383-389 e381. 2018/01/02. DOI: 10.1016/j.jacl.2017.12.001.

21. Saavedra YGL, Dufour R, Davignon J, et al. PCSK9 R46L, Lower LDL, and Cardiovascular Disease Risk in Familial Hypercholesterolemia. Arteriosclerosis, Thrombosis, and Vascular Biology 2014; 34: 2700-2705. DOI: doi:10.1161/ATVBAHA.114.304406.

22. Paquette M, Dufour R and Baass A. Scavenger Receptor LOX1 Genotype Predicts Coronary Artery Disease in Patients With Familial Hypercholesterolemia. Can J Cardiol 2017; 33: 1312-1318. 2017/09/25. DOI: 10.1016/j.cjca.2017.07.480.

23. Paquette M, Dufour R and Baass A. PHACTR1 genotype predicts coronary artery disease in patients with familial hypercholesterolemia. J Clin Lipidol 2018; 12: 966-971. 2018/05/23. DOI: 10.1016/j.jacl.2018.04.012.

24. Paquette M, Bernard S, Thanassoulis G, et al. <em >LPA </em> genotype is associated with premature cardiovascular disease in familial hypercholesterolemia. Journal of Clinical Lipidology. DOI: 10.1016/j.jacl.2019.04.006.

25. Paquette M, Chong M, Saavedra YGL, et al. The 9p21.3 locus and cardiovascular risk in familial hypercholesterolemia. J Clin Lipidol 2017; 11: 406-412. 2017/05/16. DOI: 10.1016/j.jacl.2017.01.012.

26. Futema M, Shah S, Cooper JA, et al. Refinement of variant selection for the LDL-C genetic risk score in the diagnosis of the polygenic form of clinical familial hypercholesterolemia and replication in samples from six countries. Clin Chem 2015; 61: 231-238. 
27. Hegele RA, Ban MR, Cao H, et al. Targeted next-generation sequencing in monogenic dyslipidemias. Current opinion in lipidology 2015; 26: 103-113.

28. Khera AV, Emdin CA, Drake I, et al. Genetic Risk, Adherence to a Healthy Lifestyle, and Coronary Disease. N Engl J Med 2016; 375: 2349-2358. DOI:

10.1056/NEJMoa1605086.

29. Natarajan P, Young R, Stitziel NO, et al. Polygenic Risk Score Identifies Subgroup With Higher Burden of Atherosclerosis and Greater Relative Benefit From Statin Therapy in the Primary Prevention Setting. Circulation 2017; 135: 2091-2101. 2017/02/23. DOI: 10.1161/CIRCULATIONAHA.116.024436.

30. Mega JL, Stitziel NO, Smith JG, et al. Genetic risk, coronary heart disease events, and the clinical benefit of statin therapy: an analysis of primary and secondary prevention trials. The Lancet 2015; 385: 2264-2271. DOI: https://doi.org/10.1016/S0140-6736(14)61730-X. 31. Schunkert H and Samani NJ. Statin treatment: can genetics sharpen the focus? Lancet 2015; 385: 2227-2229. 2015/03/10. DOI: 10.1016/S0140-6736(14)61931-0. 
Table 1: Baseline clinical characteristics

\begin{tabular}{lllll}
\hline Variable & Overall & $\begin{array}{l}\text { Mutation } \\
\text { Negative FH }\end{array}$ & $\begin{array}{l}\text { Mutation } \\
\text { Positive FH }\end{array}$ & $\mathbf{p}^{*}$ \\
\hline Age (years) & $49.6 \pm 13.2$ & $51.4 \pm 12.4$ & $45.4 \pm 14.0$ & $<0.001$ \\
Sex (\% Male) & $44.1 \%$ & $45.5 \%$ & $41.0 \%$ & 0.233 \\
Caucasian ethnicity (\%) & $89.6 \%$ & $92.1 \%$ & $84.1 \%$ & $<0.001$ \\
BMI (kg/m²) & $27.8 \pm 4.9$ & $28.2 \pm 4.9$ & $27.1 \pm 4.9$ & 0.007 \\
Type-2 diabetes (\%) & $6.4 \%$ & $7.0 \%$ & $5.2 \%$ & 0.337 \\
Hypertension (\%) & $30.1 \%$ & $33.2 \%$ & $23.1 \%$ & 0.004 \\
History of CAD (\%) & $25.1 \%$ & $25.0 \%$ & $25.1 \%$ & 0.987 \\
St? in therapy (\%) & $60.7 \%$ & $56.3 \%$ & $70.5 \%$ & $<0.001$ \\
Current/previous smoker (\%) & $41.9 \%$ & $42.7 \%$ & $40.2 \%$ & 0.515 \\
Systolic blood pressure & $127.5 \pm 16.3$ & $128.2 \pm 16.4$ & $125.9 \pm 16.1$ & 0.086 \\
Dis tolic blood pressure & $77.2 \pm 9.9$ & $78.2 \pm 9.8$ & $75.2 \pm 9.8$ & $<0.001$ \\
Ireatment-adjusted LDL-c (mmol/L) & $6.6 \pm 1.7$ & $6.0 \pm 1.2$ & $8.0 \pm 1.8$ & $<0.001$ \\
Triglycerides (mmol/L)\# & $1.5(1.2)$ & $1.6(1.6-1.7)$ & $1.2(1.2-1.3)$ & $<0.001$ \\
Lipoprotein(a) (g/L)\# & $0.25(0.73)$ & $0.31(0.28-0.34)$ & $0.28(0.24-0.31)$ & 0.181 \\
\hline
\end{tabular}

For continuous variables data represents the mean \pm standard deviation, and for categorical variables percentages \# Data exhibited a skewed distribution; data represents median and interquartile range or geometric mean and $95 \%$ confidence intervals. * P-value refers to comparison of means between mutation-positive and mutation-negative $\mathrm{FH}$. 
Table 2: Association between CAD GRS and clinical characteristics

\begin{tabular}{lll}
\hline Variable & $\boldsymbol{\beta}$ or OR & p \\
\hline Age (years) & $\beta=1.9(-0.3$ to 4.0$)$ & 0.089 \\
Sex (Male) & $\mathrm{OR}=1.1(0.8$ to 1.6$)$ & 0.426 \\
BMI $\left(\mathrm{kg} / \mathrm{m}^{2}\right)$ & $\beta=-0.26(-1.18$ to 0.66$)$ & 0.584 \\
Pathogenic FH mutation (Yes) & $\mathrm{OR}=0.5(0.3$ to 0.7$)$ & $<0.001$ \\
Tendon Xanthoma (Yes) & $\mathrm{OR}=0.9(0.6$ to 1.6$)$ & 0.926 \\
Type-2 diabetes (Yes) & $\mathrm{OR}=1.9(0.96$ to 3.6$)$ & 0.066 \\
Hypertension (Yes) & $\mathrm{OR}=2.0(1.4$ to 2.9$)$ & $<0.001$ \\
History of CAD (Yes) & $\mathrm{OR}=2.9(1.9$ to 4.2$)$ & $<0.001$ \\
Statin therapy (Yes) & $\mathrm{OR}=1.3(1.0$ to 1.9$)$ & 0.093 \\
Current/previous smoker $(\mathrm{Yes})$ & $\mathrm{OR}=1.1(0.8$ to 1.6$)$ & 0.509 \\
Sytolic blood pressure $(\mathrm{mm} / \mathrm{Hg})$ & $\beta=1.4(-1.4$ to 4.2$)$ & 0.331 \\
Diastolic blood pressure $(\mathrm{mm} / \mathrm{Hg})$ & $\beta=0.86(-0.83$ to 2.54$)$ & 0.319 \\
Treatment-adjusted LDL-c $(\mathrm{mmol} / \mathrm{L})$ & $\beta=-0.31(-0.58$ to -0.04$)$ & 0.025 \\
Ln triglycerides $(\mathrm{mmol} / \mathrm{L}) *$ & $\beta=0.03(-0.06$ to 0.11$)$ & 0.567 \\
Ln lipoprotein(a) $(\mathrm{g} / \mathrm{L}) *$ & $\beta=0.74(0.57$ to 0.92$)$ & $<0.001$ \\
\hline
\end{tabular}

*Data were log-transformed because of skewed distribution 
Table 3: Multivariate logistic regression analysis of the association between CAD GRS and history of CAD in mutation-positive $\mathrm{FH}$

\begin{tabular}{lccl}
\hline Variable & OR & $\mathbf{9 5 \%}$ CI & p \\
\hline Age & 1.05 & $1.02-1.08$ & $\mathbf{0 . 0 0 2}$ \\
Sex & 4.50 & $2.19-9.25$ & $<\mathbf{0 . 0 0 1}$ \\
Hypertension & 1.61 & $0.73-3.59$ & 0.239 \\
Type 2 diabetes & 1.76 & $0.45-6.92$ & 0.418 \\
Smoking & 3.56 & $1.76-7.19$ & $<\mathbf{0 . 0 0 1}$ \\
Treatment-adjusted LDL-c & 1.03 & $0.86-1.23$ & 0.754 \\
Ln Lp(a) & 1.11 & $0.79-1.55$ & 0.558 \\
CAD GRS & 3.31 & $1.34-8.17$ & $\mathbf{0 . 0 0 9}$ \\
\hline
\end{tabular}


Table 4: Multivariate logistic regression analysis of the association between CAD GRS and history of CAD in mutation-negative $\mathrm{FH}$

\begin{tabular}{lccl}
\hline Variable & OR & $\mathbf{9 5 \%}$ CI & p \\
\hline Age & 1.03 & $1.01-1.05$ & $\mathbf{0 . 0 1 0}$ \\
Sex & 2.68 & $1.65-4.36$ & $<\mathbf{0 . 0 0 1}$ \\
Hypertension & 2.87 & $1.76-4.68$ & $<\mathbf{0 . 0 0 1}$ \\
Type 2 diabetes & 1.21 & $0.51-2.88$ & 0.661 \\
Smoking & 2.07 & $1.30-3.29$ & $\mathbf{0 . 0 0 2}$ \\
Treatment-adjusted LDL-c & 0.73 & $0.59-0.91$ & $\mathbf{0 . 0 0 5}$ \\
Ln Lp(a) & 1.23 & $0.99-1.53$ & 0.058 \\
CAD GRS & 1.83 & $1.03-3.28$ & $\mathbf{0 . 0 3 9}$ \\
\hline
\end{tabular}


Table 5: Multivariate linear regression analysis of the association between CAD GRS and coronary calcium score

\begin{tabular}{llll}
\hline Variable & $\boldsymbol{\beta}$ & $\mathbf{9 5 \%}$ CI & p \\
\hline Age & 0.08 & $0.06-1.1$ & $<\mathbf{0 . 0 0 1}$ \\
Sex & 0.75 & $0.20-1.31$ & $\mathbf{0 . 0 0 8}$ \\
Hypertension & 0.85 & $0.22-1.48$ & $\mathbf{0 . 0 0 9}$ \\
Type 2 diabetes & 0.66 & $-0.66-1.98$ & 0.325 \\
Smoking & 0.26 & $-0.30-0.81$ & 0.369 \\
Treatment-adjusted LDL-c & -0.01 & $-0.18-0.16$ & 0.902 \\
Ln Lp(a) & 0.10 & $-0.14-0.34$ & 0.414 \\
Pathogenic FH mutation & 1.14 & $0.51-1.76$ & $<\mathbf{0 . 0 0 1}$ \\
CAD GRS & 0.67 & $0.03-1.31$ & $\mathbf{0 . 0 3 9}$ \\
\hline
\end{tabular}


Figure 1: Distribution of CAD GRS in mutation-negative and mutation-positive FH patients

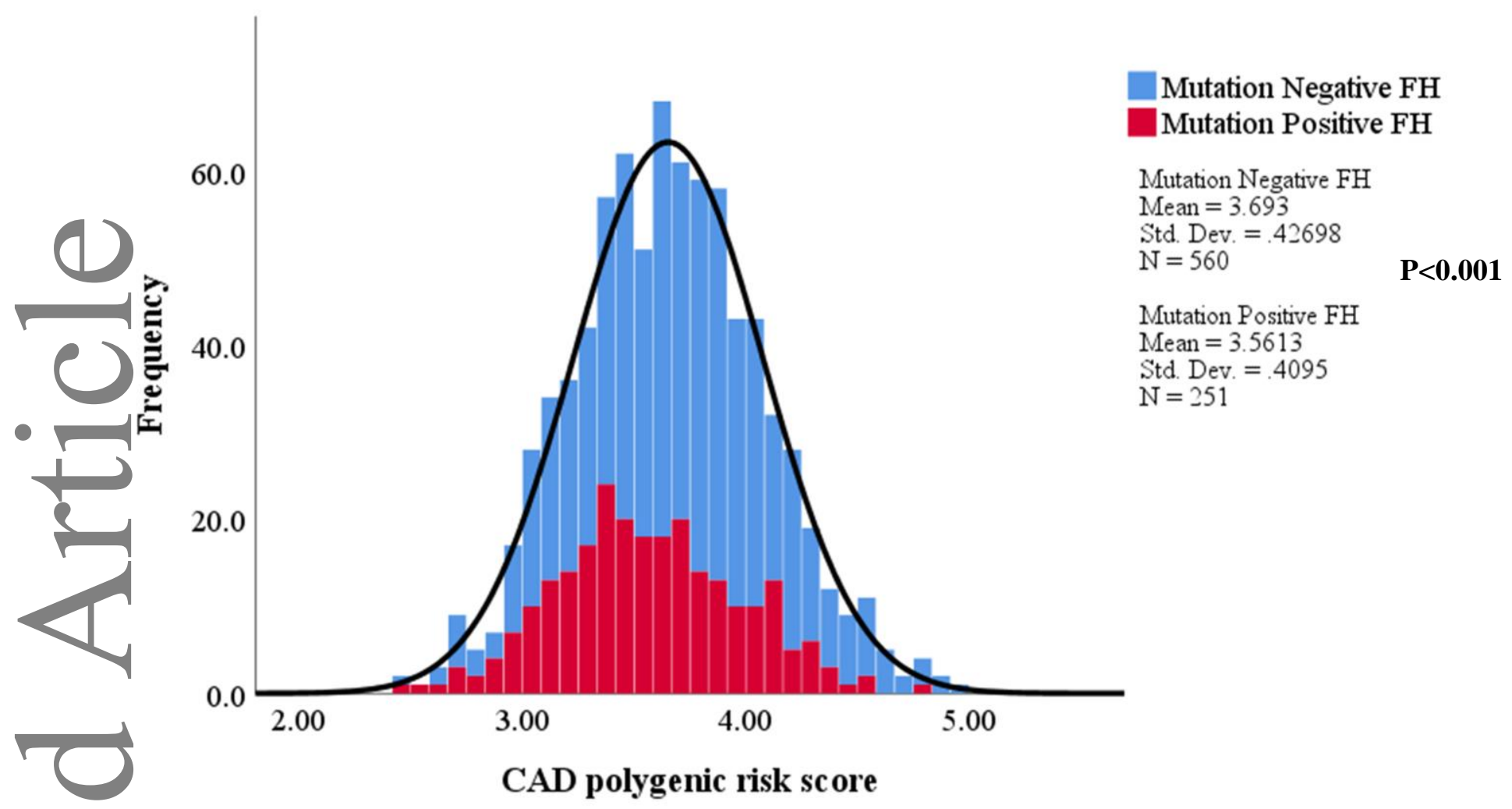




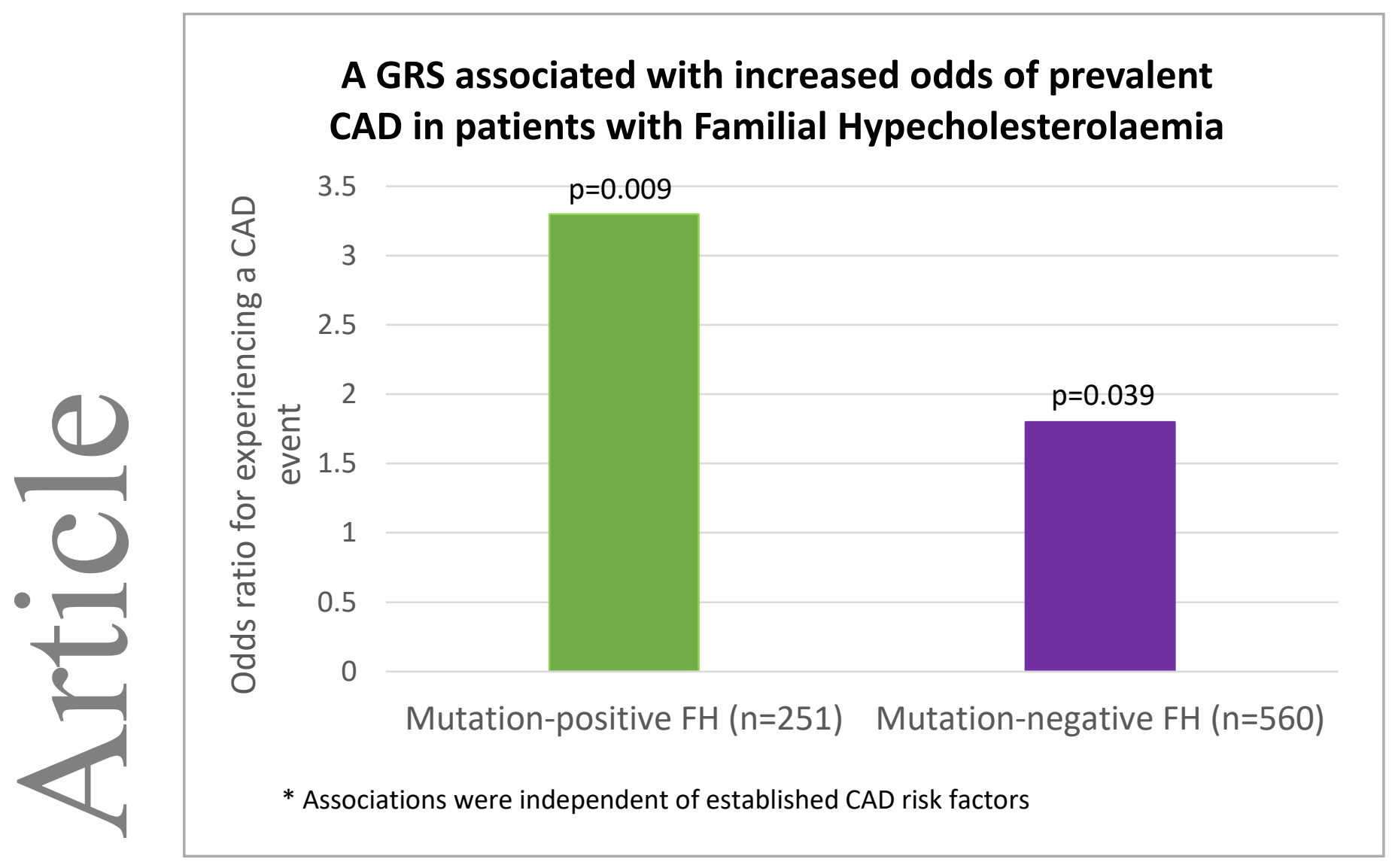

